\title{
Rule-based conflict resolution framework for Internet of Things device management in smart home environment
}

\begin{abstract}
Recent developments in Internet of Things (IoT) tremendously have introduced several heterogeneous systems and devices that characterize a smart home. Generally, these heterogeneous systems are dissimilar and accomplish various services and functionalities. Due to the gradual changes of managing resources in smart home, more heterogeneous systems are being introduced from time to time depending on the consumer requirement. As such, more dependencies are created among heterogeneous systems, and this could lead towards conflict occurrences among them. Conflicts could occur in smart home when two or more events generated by heterogeneous systems need to be triggered at an instance of time. In this paper, we present a rule-based conflict resolution framework using scheduling algorithm for managing heterogeneous systems in smart home environment. Events are captured and processed by the framework which performs corresponding conflict resolution on the heterogeneous systems. The developed framework was implemented with several heterogeneous systems to validate their effectiveness in solving conflict occurrences. The framework was ascertained to be consistent in smart home environment.
\end{abstract}

Keyword: Conflict resolution; ECA; Smart home environment 